\title{
Exclusive and Semi-inclusive $B$ Decays in QCD Factorization
}

\section{Hai-Yang Cheng*}

Institute of Physics, Academia Sinica, Taipei, Taiwan 115, R.O.C.

E-mail: phoheng@ccvax.sinica.edu.t $w_{1}$

Abstract: Applications of QCD factorization to $B \rightarrow \phi K$, charmless $B \rightarrow V V, B \rightarrow$ $J / \psi K\left(K^{*}\right)$ and semi-inclusive decays $B \rightarrow M X$ are discussed.

\section{1. $B \rightarrow \phi K$ decay}

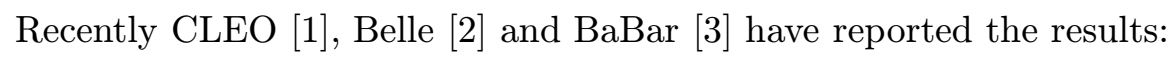

$$
\mathcal{B}\left(B^{ \pm} \rightarrow \phi K^{ \pm}\right)= \begin{cases}\left(5.5_{-1.8}^{+2.1} \pm 0.6\right) \times 10^{-6} & \text { CLEO } \\ \left(7.7_{-1.4}^{+1.6} \pm 0.8\right) \times 10^{-6} & \text { BaBar } \\ \left(11.2_{-2.0}^{+2.2} \pm 1.4\right) \times 10^{-6} & \text { Belle }\end{cases}
$$

and

$$
\mathcal{B}\left(B^{0} \rightarrow \phi K^{0}\right)= \begin{cases}<12.3 \times 10^{-6} & \text { CLEO } \\ \left(8.1_{-2.5}^{+3.1} \pm 0.8\right) \times 10^{-6} & \text { BaBar } \\ \left(8.9_{-2.7}^{+3.4} \pm 1.0\right) \times 10^{-6} & \text { Belle }\end{cases}
$$

The neutral mode $B^{0} \rightarrow \phi K^{0}$ is a pure penguin process, while the charged mode $\phi K^{-}$ receives an additional annihilation contribution which is quark-mixing-angle suppressed. The predicted branching ratio is very sensitive to the nonfactorizable effects which are sometimes parameterized in terms of the effective number of colors $N_{c}^{\text {eff }}$; it falls into a broad range $(13 \sim 0.4) \times 10^{-6}$ for $N_{c}^{\text {eff }}=2 \sim \infty\left[\begin{array}{l}{[\overline{4}} \\ i\end{array}\right]$. Therefore, a theory calculation of the nonfactorizable corrections is urgently needed in order to have a reliable prediction which can be used to compare with experiment.

In QCD factorization approach, the branching ratio is predicted to be $\mathcal{B}\left(B^{ \pm} \rightarrow\right.$ $\left.\phi K^{ \pm}\right)=(4.0 \pm 0.8) \times 10^{-6}$ in the absence of annihilation contributions, where theoretical error comes from the logarithmic divergent term occurring in spectator interactions

$$
X_{H} \equiv \int_{0}^{1} \frac{d x}{x}=\ln \frac{M_{B}}{\Lambda_{\mathrm{QCD}}}\left(1+\rho_{H}\right), \quad \rho_{H} \leq 1
$$

\footnotetext{
${ }^{*}$ Speaker.
} 
Power-suppressed annihilation is often treated to be negligible based on helicity suppression argument. However, annihilation diagrams induced by $(S-P)(S+P)$ penguin operators are not subject to helicity suppression. Including theoretical errors from both $X_{H}$ for

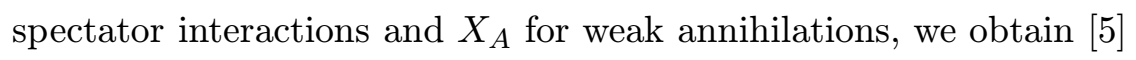

$$
\mathcal{B}\left(B^{-} \rightarrow \phi K^{-}\right)=\left(4.6_{-1.5}^{+3.2}\right) \times 10^{-6}, \quad \mathcal{B}\left(B^{0} \rightarrow \phi K^{0}\right)=\left(4.2_{-1.3}^{+3.0}\right) \times 10^{-6} .
$$

Hence, the prediction is in agreement with data within experimental and theoretical errors.

Recently, calculations within the framework of pQCD are also available [i] results $\mathcal{B}\left(B^{-} \rightarrow \phi K^{-}\right)=\left(10.2_{-2.1}^{+3.9}\right) \times 10^{-6}$ and $\mathcal{B}\left(B^{0} \rightarrow \phi K^{0}\right)=\left(9.6_{-2.0}^{+3.7}\right) \times 10^{-6}$ are large for two reasons. First, the relevant scale in the pQCD calculation is $\mu \sim 1.5 \mathrm{GeV}$ and the relevant Wilson coefficient $c_{4}(\mu)$ at this low scale increases dramatically as $\mu$ decreases. However, such a "dynamic enhancement" does not exist in QCD factorization because the parameter $a_{4}$ which contains the term $c_{4}(\mu)+c_{3}(\mu) / 3$ is formally renormalization scale and $\gamma_{5}$ scheme independent after including $\mathcal{O}\left(\alpha_{s}\right)$ vertex-type and penguin-type corrections. Second, the contribution from the chromomagnetic dipole operator to $a_{4}$, which is absent in the current pQCD calculation, is sizable but destructive. Therefore, a refined measurement of $B \rightarrow \phi K$ decays will provide a nice ground for discriminating between the approaches of QCD factorization and $\mathrm{pQCD}$.

\section{Charmless $B \rightarrow V V$ decays}

It is known that the decay amplitude of a $B$ meson into two vector mesons is governed by three unknown form factors $A_{1}\left(q^{2}\right), A_{2}\left(q^{2}\right)$ and $V\left(q^{2}\right)$ in the factorization approach. It has been pointed out in [i] that the charmless $B \rightarrow V V$ rates are very sensitive to the form-factor ratio $A_{2} / A_{1}$. This form-factor ratio is almost equal to unity in the Bauer-

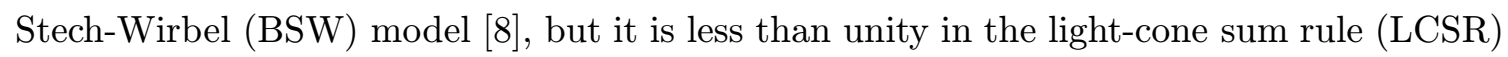
analysis for form factors [9. the LCSR are always larger than that by the BSW model by a factor of $1.6 \sim 2\left[\begin{array}{l}{[\mathbf{7}} \\ ]\end{array}\right]$. This is understandable because in the heavy quark limit, both vector mesons in the charmless $B \rightarrow V V$ decay should have zero helicity and the corresponding amplitude is proportional to the form factor difference $\left(A_{1}-A_{2}\right)$. These two form factors are identical at $q^{2}=0$ in the BSW model.

We have analyzed $B \rightarrow V V$ decays within the framework of QCD factorization [i] 1010.1$]$. We see from Table I that the first observed charmless $B \rightarrow V V$ mode, $B \rightarrow \phi K^{*}$, recently measured by CLEO [i], Belle [2] and BaBar [i3i], clearly favors the LCSR over the BSW model for $B-V$ transition form factors. Contrary to phenomenological generalized factorization, nonfactorizable corrections to each partial-wave or helicity amplitude are not the same; the effective parameters $a_{i}$ vary for different helicity amplitudes. The leading-twist nonfactorizable corrections to the transversely polarized amplitudes vanish in the chiral limit and hence it is necessary to take into account twist-3 distribution amplitudes of the vector meson in order to have renormalization scale and scheme independent predictions. Owing to the absence of $(S-P)(S+P)$ penguin operator contributions to $W$-emission amplitudes, tree-dominated $B \rightarrow V V$ decays tend to have larger branching ratios than the 


\begin{tabular}{|l|lll|}
\hline Decay & LCSR & BSW & Expt. \\
\hline$B^{-} \rightarrow K^{*-} \phi$ & 9.30 & 4.32 & $9.7_{-3.4}^{+4.2} \pm 1.7$ (BaBar) \\
& & & $10.6_{-4.9-1.6}^{+6.4+1.8}$ (CLEO) \\
& & & $<18$ (Belle) \\
\hline $\bar{B}^{0} \rightarrow \bar{K}^{* 0} \phi$ & 8.71 & 4.62 & $8.6_{-2.4}^{+2.8} \pm 1.1$ (BaBar) \\
& & & $11.5_{-3.7-1.7}^{+4.5+1.8}(\mathrm{CLEO})$ \\
& & & $13.0_{-5.2}^{+6.4} \pm 2.1$ (Belle) \\
\hline
\end{tabular}

Table 1: Branching ratios (in units of $10^{-6}$ ) for $B \rightarrow K^{*} \phi$ modes. Two different form-factor models, the LCSR and the BSW models, are adopted and the unitarity angle $\gamma=60^{\circ}$ is employed.

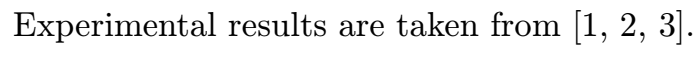

penguin-dominated ones [1] ${ }_{1}^{1}$ in]. For example, $B^{0} \rightarrow \rho^{+} \rho^{-}$has a branching ratio of order $4 \times 10^{-5}$.

\section{3. $B \rightarrow J / \psi K\left(K^{*}\right)$ decays}

The hadronic decays $B \rightarrow J / \psi K\left(K^{*}\right)$ are interesting because experimentally they are a few of the color-suppressed modes which have been measured, and theoretically they are calculable by QCD factorization even the emitted meson $J / \psi$ is heavy. That is, this is the only color-suppressed mode that one can compute and compare with experiment.

To leading-twist contributions from the light-cone distribution amplitudes (LCDAs) of the mesons, vertex corrections and hard spectator interactions including $m_{c}$ effects imply $\left|a_{2}(J / \psi K)\right| \sim 0.11$ vs. 0.25 by experiment [i] $\left.i_{2}^{1} i_{1}\right]$. Hence, the predicted branching ratio is too small by a factor of 5 ; the nonfactorizable corrections to naive factorization to leading-twist order are small. We study the twist-3 effects due to the kaon and find that the coefficient $a_{2}(J / \psi K)$ is largely enhanced by the nonfactorizable spectator interactions arising from the twist-3 kaon LCDA $\phi_{\sigma}^{K}$, which are formally power-suppressed but chirally, logarithmically and kinematically enhanced. Therefore, factorization breaks down at twist-3 order. It is found in [i $\left.{ }_{1}^{1} i_{1}^{\prime}\right]$ that $a_{2}(J / \psi K)=0.19_{-0.12}^{+0.14}$ for $\left|\rho_{H}\right| \leq 1$ and that twist-2 as well as twist-3 hard spectator interactions are equally important.

Recently, the spin amplitudes $A_{0}, A_{\|}$and $A_{\perp}$ for $B \rightarrow J / \psi K^{*}$ decays in the transversity

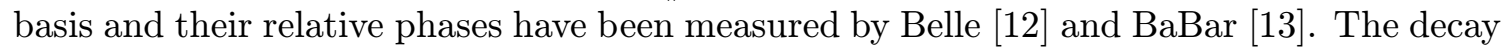
$B \rightarrow J / \psi K^{*}$ is currently analyzed within the framework of QCD factorization [1] is found that the effective parameters $a_{2}^{h}$ for helicity $h=0,+,-$ states receive different nonfactorizable contributions. Contrary to the $J / \psi K$ case, $a_{2}^{0}$ in $B \rightarrow J / \psi K^{*}$ does not receive twist-3 contributions and it is dominated by twist-2 hard spectator interactions.

\section{Semi-inclusive $B$ decays}

The semi-inclusive decays $B \rightarrow M+X$ that are of special interest originate from the quark level decay, $b \rightarrow M+q$. They are theoretically cleaner compared to exclusive decays and

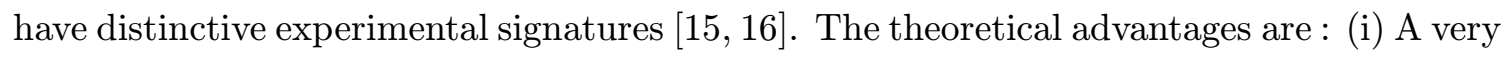


important theoretical simplification occurs in the semi-inclusive decays over the exclusive decays if we focus on final states such that $M$ does not contain the spectator quark of the decaying $B\left(B_{s}\right)$ meson as then we completely by-pass the need for the transition form factor for $B\left(B_{s}\right) \rightarrow M$. (ii) There is no troublesome infrared divergent problem occurred at endpoints when working in QCD factorization, contrary to the exclusive decays where endpoint infrared divergences usually occur at twist-3 level, and (iii) As for $C P$ violation, contrary to the exclusive hadronic decays, it is not plagued by the unknown soft phases. Consequently, the predictions of the branching ratios and partial rate asymmetries for $B \rightarrow M X$ are considerably clean and reliable. Since these semi-inclusive decays also tend to have appreciably larger branching ratios compared to their exclusive counterparts, they may therefore be better suited for extracting CKM-angles and for testing the Standard Model.

In order to have a reliable study of semi-inclusive decays both theoretically and experimentally, we will impose two cuts. First, a momentum cutoff imposed on the emitted light meson $M$, say $p_{M}>2.1 \mathrm{GeV}$, is necessary in order to reduce contamination from the unwanted background and ensure the relevance of the two-body quark decay $b \rightarrow M q$. Second, it is required that the meson $M$ does not contain the spectator quark in the initial $B$ meson and hence there us no $B-M$ transition form factors. Under these two cuts, we argue that the factorization formula for exclusive decays can be generalized to the semi-inclusive decay:

$$
\langle M X|O| B\rangle=\underbrace{\int_{0}^{1} d u T^{I}(u) \Phi_{M}(u)}_{\text {no form factors }}+\underbrace{\int_{0}^{1} d \xi d u T^{I I}(\xi, u) \Phi_{B}(\xi) \Phi_{M}(u)}_{\text {power suppressed by }\left(\Lambda_{\mathrm{QCD}} / m_{b}\right)^{1 / 2}} .
$$

However, this factorization formula is not as rigorous as the one for the exclusive case. To the order $\mathcal{O}\left(\alpha_{s}\right)$, there are two additional contributions besides vertex corrections: the bremsstrahlung process $b \rightarrow M q g$ ( $g$ being a real gluon) and the process $b \rightarrow M q g^{*} \rightarrow$ $M q q^{\prime} \bar{q}^{\prime}$. The bremsstrahlung subprocess could potentially suffer from the infrared divergence. However, the vertex diagram in which a virtual gluon is attached to $b$ and $q$ quarks is also infrared divergent. This together with the above-mentioned bremsstrahlung process will lead to a finite and well-defined correction. This finite correction is expected to be small as it is suppressed by a factor of $\alpha_{s} / \pi \approx 7 \%$. In the presence of bremsstrahlung and the fragmentation of the quark-antiquark pair from the gluon, the factorizable configurations $\left\langle X_{1} M\left|j_{1}\right| 0\right\rangle\left\langle X_{1}^{\prime}\left|j_{2}\right| B\right\rangle$ and $\left\langle X_{2}\left|j_{1}\right| 0\right\rangle\left\langle X_{2}^{\prime} M\left|j_{2}\right| B\right\rangle$ with $X_{1}+X_{1}^{\prime}=X$ and $X_{2}+X_{2}^{\prime}=X$ are allowed. In general, one may argue that these configurations are suppressed since the momentum cut $p_{M}>2.1 \mathrm{GeV}$ favors the two-body quark decay $b \rightarrow M q$ and low multiplicity for $X$. However, it is not clear to us how rigorous this argument is. Therefore, we will confine ourselves to vertex-type and penguin-type corrections as well as hard spectator interactions so that the factorization formula $\left(\begin{array}{l}1 \\ \mathbf{4}\end{array} \overline{1}_{\mathbf{2}}^{\prime}\right)$ is applicable to semi-inclusive decays at least as an approximation.

Some highlights of the present analysis are [i] $\left.\overline{1}_{1}\right]$ : 
- Though phase-space and power suppressed, hard spectator interactions are extremely important for color-suppressed modes, e.g. $\left(\pi^{0}, \rho^{0}, \omega\right) X_{\bar{s}}, \phi X$ and $J / \psi X_{s}, J / \psi X$. This is because the relevant hard spectator correction is color allowed, whereas $b \rightarrow$ $M q$ for these modes are color-suppressed.

- The prediction $\mathcal{B}\left(B \rightarrow J / \psi X_{s}\right)=9.6 \times 10^{-3}$ is in agreement with experiments: $(8.0 \pm 0.8) \times 10^{-3}$ from CLEO and $(7.89 \pm 0.10 \pm 0.40) \times 10^{-3}$ from BaBar.

- $\bar{B}_{s}^{0} \rightarrow\left(\pi^{0}, \rho^{0}, \omega\right) X_{\bar{s}}, \rho^{0} X_{s \bar{s}}, \bar{B}^{0} \rightarrow\left(K^{-} X, K^{*-} X\right)$ and $B^{-} \rightarrow\left(K^{0} X_{s}, K^{* 0} X_{s}\right)$ are the most promising ones in searching for direct $\mathrm{CP}$ violation: they have branching ratios of order $10^{-6}-10^{-4}$ and $\mathrm{CP}$ rate asymmetries of order $(10-40) \%$. With $1 \times 10^{7}$ $B \bar{B}$ pairs, the asymmetry in $K^{*-}$ channel starts to become accessible. With about $7 \times 10^{7} B \bar{B}$ events, the PRA's in other modes mentioned above will become feasible.

\section{Acknowledgments}

I wish to thank Kwei-Chou Yang and Amarjit Soni for collaborations and Physics Department, Brookhaven National Laboratory for its hospitality.

\section{References}

[1] CLEO Collaboration, R.A. Briere et al., hep-ex/0101032.

[2] Belle Collaboration, A. Bozek, this proceedings and BELLE-CONF-0113.

[3] BaBar Collaboration, B. Aubert et al., hep-ex/0105001.

[4] H.Y. Cheng and K.C. Yang. Phys. Rev. D62 (2000) 054029.

[5] H.Y. Cheng and K.C. Yang, Phys. Rev. D64 (2001) 074004.

[6] C.H. Chen, Y.Y. Keum, and H.n. Li, hep-ph/0107165; S. Mishima, hep-ph/0107163 and hep-ph/0107206.

[7] Y.H. Chen, H.Y. Cheng, B. Tseng, and K.C. Yang, Phys. Rev. D60 (1999) 094014.

[8] M. Wirbel, B. Stech, and M. Bauer, Z. Phys. C29 (1985) 637; M. Bauer, B. Stech, and M. Wirbel, Z. Phys. C34 (1987) 103.

[9] P. Ball and V.M. Braun, Phys. Rev. D58 (1998) 094016; P. Ball, J. High Energy Phys. 09 (1998) 005 [hep-ph/9802394].

[10] H.Y. Cheng and K.C. Yang, Phys. Lett. B511 (2001) 40.

[11] H.Y. Cheng and K.C. Yang, Phys. Rev. D63 (2001) 074011; J. Chay and C. Kim, hep-ph/0009244.

[12] Belle Collaboration, K. Abe et al. BELLE-CONF-0105.

[13] BaBar Collaboration, B. Aubert et al., hep-ex/0107049.

[14] H.Y. Cheng, Y.Y. Keum, and K.C. Yang, in preparation.

[15] D. Atwood and A. Soni, Phys. Rev. Lett. 81 (1998) 3324.

[16] T.E. Browder, A. Datta, X.G. He, and S. Pakvasa, Phys. Rev. D57 (1998) 6829.

[17] H.Y. Cheng and A. Soni, hep-ph/0105246, to appear in Phys. Rev. D. 